\title{
BOUNDARY ELEMENT ANALYSIS OF ANISOTROPIC THERMOMAGNETOELECTROELASTIC SOLIDS WITH 3D SHELL-LIKE INCLUSIONS
}

\author{
Iaroslav PASTERNAK*, Heorhiy SULYM* \\ *Department of Computer Engineering, Lutsk National Technical University, Lvivska Str 75, 43018 Lutsk, Ukraine \\ *Department of Mechanics and Applied Computer Science, Bialystok University of Technology, ul. Wiejska 45C, 15-351 Bialystok, Poland \\ yaroslav.pasternak@gmail.com, h.sulym@pb.edu.pl
}

received 21 April 2017, revised 5 December 2017, accepted 8 December 2017

\begin{abstract}
The paper presents novel boundary element technique for analysis of anisotropic thermomagnetoelectroelastic solids containing cracks and thin shell-like soft inclusions. Dual boundary integral equations of heat conduction and thermomagnetoelectroelasticity are derived, which do not contain volume integrals in the absence of distributed body heat and extended body forces. Models of 3D soft thermomagnetoelectroelastic thin inclusions are adopted. The issues on the boundary element solution of obtained equations are discussed. The efficient techniques for numerical evaluation of kernels and singular and hypersingular integrals are discussed. Nonlinear polynomial mappings are adopted for smoothing the integrand at the inclusion's front, which is advantageous for accurate evaluation of field intensity factors. Special shape functions are introduced, which account for a square-root singularity of extended stress and heat flux at the inclusion's front. Numerical example is presented.
\end{abstract}

Key words: Anisotropic, 3D, Thermomagnetoelectroelastic, Crack, Thin Inclusion

\section{INTRODUCTION}

Rapid development of modern multi-field materials and microelectro-mechanical technologies raises increasing attention to their modeling and simulation. Particular interest is focused on the issues of fracture mechanics of cracked and inhomogeneous thermomagnetoelectroelastic (TMEE) materials. Since TMEE materials are anisotropic by nature, their analysis is more complicated than those of isotropic materials.

The boundary element method (BEM) is widely applied in the crack analysis (Aliabadi, 1997), since it allows accurate evaluation of field intensity factors at the crack front and requires only boundary mesh. Various boundary element techniques were proposed for 3D crack analysis in multi-field materials (e.g. see Pan and Yuan (2000), Rungamornrat et al. (2015), Muñoz-Reja et al. (2016)). Nevertheless, only recently (Pasternak et al., 2017) it was developed general 3D BEM for analysis of 3D cracks in anisotropic medium, which couples both thermal and magnetoelectro-mechanical fields.

Another issue is the analysis of thin $3 D$ inclusions. Various techniques are introduced to study soft or rigid inhomogeneities; however, they are mainly limited to the cases of planar perfectly rigid inclusions (Mykhas'kiv et al. (2010), Selvadurai (2000, 2002)). Also the models of thin inclusions should be accounted for in the modeling of semi-permeable cracks.

Therefore, this paper utilizes previously developed dual boundary integral equations (Pasternak et al., 2017) for obtaining the dual BEM for TMEE solids containing discontinuity surfaces (heat flux, stress, electric displacement, magnetic induction, temperature, displacement, electric and magnetic potentials discontinuity). Models of soft semi-permeable shell-like inclusions are introduced and incorporated in the obtained integral equations for obtaining the solution to $3 \mathrm{D}$ crack and inclusion problems. The issues on the efficient numerical evaluation of kernel functions, integration of singular and hypersingular integrals and accurate determination of field intensity factors are discussed in details.

\section{GOVERNING EQUATIONS OF HEAT CONDUCTION AND THERMOMAGNETOELECTROELASTICITY}

According to Pasternak et al. (2017), in a fixed Cartesian coordinate system $O x_{1} x_{2} x_{3}$ the equilibrium equations, the Maxwell equations (Gauss theorem for electric and magnetic fields), and the balance equations of heat conduction in the steady-state case can be presented in the following compact form

$\tilde{\sigma}_{I j, j}+\tilde{f}_{I}=0, h_{i, i}-f_{h}=0$,

where the capital index varies from 1 to 5 , while the lower case index varies from 1 to 3 , i.e. $I=1,2, \ldots, 5 . i=1,2,3$. Here and further the Einstein summation convention is used. A comma at subscript denotes differentiation with respect to a coordinate indexed after the comma, i.e. $u_{i, j}=\partial u_{i} / \partial x_{j}$.

In the assumption of small strains and fields' strengths the constitutive equations of linear thermomagnetoelectroelasticity in the compact notations are as follows (Pasternak et al., 2017):

$\tilde{\sigma}_{j}=\tilde{C}_{I j K m} \tilde{u}_{K, m}-\tilde{\beta}_{I j} \theta, h_{i}=-k_{i j} \theta_{, j}$,

where:

$\tilde{u}_{i}=u_{i}, \tilde{u}_{4}=\phi, \tilde{u}_{5}=\psi ; \tilde{f}_{i}=f_{i}, \tilde{f}_{4}=-q, \tilde{f}_{5}=b_{m} ;$
$\tilde{\sigma}_{i j}=\sigma_{i j}, \tilde{\sigma}_{4 j}=D_{j}, \tilde{\sigma}_{5 j}=B_{j} ;$
$\tilde{C}_{i j k m}=C_{i j k m}, \tilde{C}_{i j 4 m}=e_{m i j}, \tilde{C}_{4 j k m}=e_{j k m}, \tilde{C}_{4 j 4 m}=-\kappa_{j m}$,$$
\tilde{C}_{i j k m}=C_{i j k m}, \widetilde{C}_{i j 4 m}=e_{m i j}, \tilde{C}_{4 j k m}=e_{j k m}, \tilde{C}_{4 j 4 m}=-\kappa_{j m},
$$ 
$\tilde{C}_{i j 5 m}=h_{m i j}, \tilde{C}_{5 j k m}=h_{j k m}, \tilde{C}_{5 j 5 m}=-\mu_{j m^{\prime}} \tilde{\beta}_{i j}=\beta_{i j}{ }^{\prime}$
$\tilde{C}_{4 j 5 m}=-\gamma_{j m}, \widetilde{C}_{5 j 4 m}=-\gamma_{j m} ;$

$\tilde{\beta}_{4 j}=-\chi_{j}, \tilde{\beta}_{5 j}=v_{j}(i, j, k, m=1,2,3)$;

$\sigma_{i j}$ is a stress tensor; $f_{i}$ is a body force vector; $D_{i}$ is an electric displacement vector; $q$ is a free charge volume density; $B_{i}$ is a magnetic induction vector; $b_{m}$ is a body current; $h_{i}$ is a heat flux; $f_{h}$ is a distributed heat source density; $u_{i}$ is a displacement vector; $\phi, \psi$ are the electric and magnetic potentials, respectively; $\theta$ is a temperature change with respect to the reference temperature; $C_{i j k m}$ are the elastic stiffnesses (elastic moduli); $k_{i j}$ are heat conduction coefficients; $e_{i j k}, h_{i j k}$ are piezoelectric and piezomagnetic constants; $\kappa_{i j}, \mu_{i j}, \gamma_{i j}$ are dielectric permittivities, magnetic permeabilities and electromagnetic constants, respectively; $\beta_{i j}, \chi_{i}$ and $v_{i}$ are thermal moduli, pyroelectric coefficients and pyromagnetic coefficients, respectively.

Thus, the problem of linear thermomagnetoelectroelasticity is to solve partial differential equations (1) and (2) under the given boundary conditions and volume loading. Since magneto-electromechanical fields do not influence temperature field in the considered problem (uncoupled thermomagnetoelectroelasticity) the first step is to solve the heat conduction equation and the second one is to determine mechanical, electric and magnetic fields acting in the solid.

\section{DUAL BOUNDARY INTEGRAL EQUATIONS AND MODELS OF 3D SOFT SHELL-LIKE INCLUSIONS}

For modeling of solids with thin inhomogeneities, a coupling principle for continua of different dimension is often used (Sulym, 2007). This principle involves the replacement of a thin inclusion with a surface of a field discontinuity for stress, electric displacement, displacement, electric potential, temperature, and heat flux. Frequently a median surface of a thin inhomogeneity is chosen as the discontinuity surface. The inclusion is thus removed from consideration as a geometrical object, and it is assumed that the thermal, electric and mechanical influence of the inclusion is reduced to the influence of the above-mentioned discontinuity surface (Fig. 1). Thus, according to a discontinuity function method (Sulym, 2007), the study of a stress state of a solid (an exterior problem) is reduced to the study of the influence of unknown discontinuity functions and is considered without account of the inclusion's material properties. It is clear that the thermoelectroelastic state of the solid depends on these discontinuity functions, material properties of the solid, the geometrical features of the problem, the contact conditions at the thin inhomogeneity interface, and the external load.

On the other hand, due to a small thickness of the inclusion, the extended tractions and displacements, temperature and heat flux at the faces of the inclusion are related with each other. Corresponding relations, which include thermo-magneto-electromechanical properties of the inclusion and its thickness, are called the mathematical model of a thin inclusion. This model does not depend on the properties of a medium containing an inclusion, and it can be considered as an interior problem. There are only three basic requirements for the mathematical model of a thin inclusion (Sulym, 2007): (a) the number of equations should equal to the number of the unknown discontinuity functions; (b) the model should be simple for further solution of the obtained system of equations; and (c) the model should simulate essential features of thermoelectroelastic behavior of the inclusion.
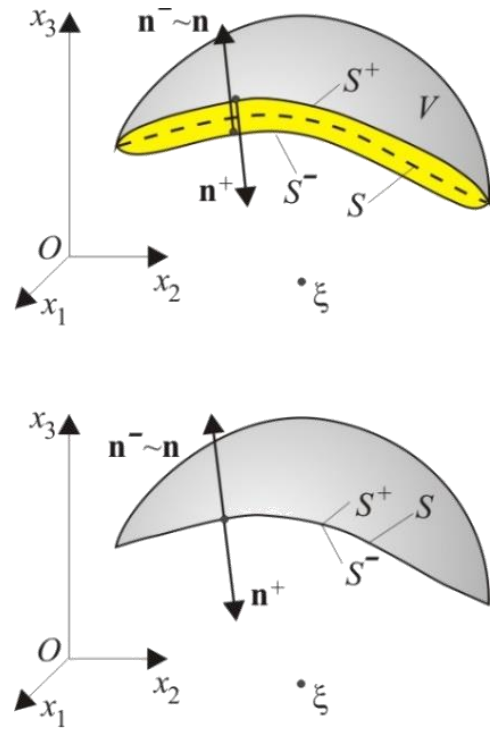

Fig. 1. Simplification of a thin inclusion

Since the coupling principle and a discontinuity function method consider exterior and interior problems independently, several inclusion models, which simulate different features of the inhomogeneity, can be developed for the same exterior problem, and using the same inclusion model one can solve different exterior problems.

According to the philosophy of the discontinuity function approach the exterior problem of 3D TMEE is reduced to the following system of dual boundary integral equations (see Pasternak et al. (2017)):

- heat conduction

$$
\begin{gathered}
\frac{1}{2} \Sigma \theta\left(\mathbf{x}_{0}\right)=\iint_{S} \Theta^{*}\left(\mathbf{x}, \mathbf{x}_{0}\right) \Sigma h_{n}(\mathbf{x}) d S(\mathbf{x}) \\
-\operatorname{CPV} \iint_{S} H^{*}\left(\mathbf{x}, \mathbf{x}_{0}\right) \Delta \theta(\mathbf{x}) d S(\mathbf{x}), \\
\frac{1}{2} \Delta h_{n}\left(\mathbf{x}_{0}\right)=n_{i}\left(\mathbf{x}_{0}\right)\left[\operatorname{CPV} \iint_{S} \Theta_{i}^{* *}\left(\mathbf{x}, \mathbf{x}_{0}\right) \Sigma h_{n}(\mathbf{x}) d S(\mathbf{x})\right. \\
\left.-\operatorname{HFP} \iint_{S} H_{i}^{* *}\left(\mathbf{x}, \mathbf{x}_{0}\right) \Delta \theta(\mathbf{x}) d S(\mathbf{x})\right],
\end{gathered}
$$

- TMEE

$$
\begin{aligned}
& \frac{1}{2} \Delta \tilde{t}_{I}\left(\mathbf{x}_{0}\right)=n_{j}\left[\operatorname{CPV} \iint_{S} D_{I j K}\left(\mathbf{x}, \mathbf{x}_{0}\right) \Sigma \tilde{t}_{K}(\mathbf{x}) d S(\mathbf{x})\right. \\
& -\mathrm{HFP} \iint_{S} S_{I j K}\left(\mathbf{x}, \mathbf{x}_{0}\right) \Delta \tilde{u}_{K}(\mathbf{x}) d S(\mathbf{x}) \\
& \quad+\operatorname{CPV} \iint_{S} Q_{I j}\left(\mathbf{x}, \mathbf{x}_{0}\right) \Delta \theta(\mathbf{x}) d S(\mathbf{x}) \\
& \left.\quad+\iint_{S} W_{I j}\left(\mathbf{x}, \mathbf{x}_{0}\right) \Sigma h_{n}(\mathbf{x}) d S(\mathbf{x})\right],
\end{aligned}
$$

where $\Sigma \mathrm{f}=\mathrm{f}^{+}+\mathrm{f}^{-} ; \Delta \mathrm{f}=\mathrm{f}^{+}-\mathrm{f}^{-} ; \mathrm{n}_{\mathrm{p}}$ is a unit outwards normal vector to the surface $\mathrm{S}$ (or its faces $\mathrm{S}^{+}$and $\mathrm{S}^{-}$), which replaces an inclusion; $\tilde{\mathrm{t}}_{\mathrm{I}}=\widetilde{\sigma}_{\mathrm{Ij}} \mathrm{n}_{\mathrm{j}}$ is an extended traction vector; $\mathrm{h}_{\mathrm{n}}=\mathrm{h}_{\mathrm{i}} \mathrm{n}_{\mathrm{i}}$; CPV stands for the Cauchy Principal Value of the integral, and HFP stands for the Hadamard Finite Part of the integral. All the kernels are given explicitly by Pasternak et al. (2017). 
The interior problem is then solved as follows. Using the philosophy of the discontinuity function method and the coupling principle (Fig. 1) for the development of a shell-like inclusion model, one withdraws the inclusion from consideration as a geometrical object, and transfers the contact tractions, displacements, surface charges, electric potential, temperature, and heat flux onto its median surface $\mathrm{S}$ (accordingly onto the faces $\mathrm{S}^{+}$and $\mathrm{S}^{-}$, Fig. 1). Thus, the problem is reduced to the determination of the thermomagnetoelectroelastic state of a solid with the surface $S$ of thermo-magneto-electro-mechanical field discontinuities. After development of the interaction conditions for a thin inhomogeneity along with the integral equations (4)-(5) concerning abovementioned field discontinuities for a solid, the thermoelectroelastic state of the latter can be determined.

Consider the thermomagnetoelectroelastic state at a certain point $y$ on the median surface of a thin transversely isotropic pyroelectric inclusion. Assume that the $\mathrm{Ox}_{3}^{\prime}$ axis of a local coordinate system $\mathrm{Ox}_{1}{ }_{1} \mathrm{x}_{2}{ }_{2} \mathrm{x}_{3}$ is directed along the normal vector $\mathrm{n}^{+}(\mathrm{y})$ of the surface $\mathrm{S}^{+}$at the point $\mathrm{y}$. All vectors in the local coordinate system are related with those of the global one by the relations $\tilde{\mathrm{t}}^{\prime} \quad \mathrm{I}=\Omega \mathrm{IJ} \tilde{\mathrm{t} J}, \tilde{\mathrm{t}}_{\mathrm{I}}=\Omega_{\mathrm{JI}} \tilde{\mathrm{t}}_{\mathrm{J}}^{\prime}$, where $\Omega$ is a rotation matrix. The origin of the local coordinate system is placed at the median surface of the thin inclusion. Thus, $\tilde{\mathrm{f}}(-\mathrm{h})=\tilde{\mathrm{f}}^{+}$and $\tilde{\mathrm{f}}(\mathrm{h})=\tilde{\mathrm{f}}^{-}$, where $\tilde{\mathrm{f}}$ is one of the scalars $\theta, \mathrm{h}_{\mathrm{n}}$ or vectors $\tilde{\mathrm{u}}_{\mathrm{i}}, \tilde{\mathrm{t}}_{\mathrm{i}} ; 2 \mathrm{~h}=2 \mathrm{~h}(\mathrm{y})$ is the thickness of the inclusion at $y$. With the account of the identity $\mathrm{n}^{ \pm}=-\mathrm{n}^{\mathrm{i} \pm}$, the conditions of a perfect thermal, mechanical, magnetic and electric contact of the inclusion and the solid are $\theta^{ \pm}=\theta^{i \pm}, h_{n}^{ \pm}=-h_{n}^{i \pm}, \tilde{u}_{i}^{ \pm}=\tilde{u}_{i}^{i \pm}, \tilde{\mathrm{t}}_{i}^{ \pm}=-\tilde{\mathrm{t}}_{i}^{i^{ \pm}}$. Here the non-italic superscript " $\mathrm{i}$ " denotes values concerned with the inclusion.

According to Eq. (2), heat flux and extended stress inside the inclusion in the local coordinate system equal

$$
h_{3}^{\prime}=-k_{3 j}^{\prime \mathrm{i}} \theta_{, j}^{\prime} ; \quad \widetilde{\sigma}^{\prime} \quad I 3=C_{I 3 K m}^{\prime^{\mathrm{i}}} \underset{u^{\prime}}{\widetilde{2}} K, m-\widetilde{\beta}^{\prime} I 3 \mathrm{i} \theta .
$$

Integrating Eq. (6) over the thickness of the inclusion one can obtain

$$
\begin{aligned}
& \int_{-h}^{h} h_{3}^{\prime} d x_{3}^{\prime}=-k_{33}^{\prime \mathrm{i}}[\theta(h)-\theta(-h)] \\
& \quad-k_{31}^{\prime \mathrm{i}} \int_{-h}^{h} \theta_{, 1}^{\prime} d x_{3}^{\prime}-k_{32}^{\prime \mathrm{i}} \int_{-h}^{h} \theta_{, 2}^{\prime} d x_{3}^{\prime}, \\
& \int_{-h}^{h} \tilde{\sigma}_{I 3}^{\prime} d x_{3}^{\prime}=C_{I 3 K 3}^{\mathrm{i}}\left[\tilde{u}_{K}^{\prime}(h)-\tilde{u}_{K}^{\prime}(-h)\right] \\
& \quad+C_{I 3 K 1}^{\prime \mathrm{i}} \int_{-h}^{h} \tilde{u}_{K, 1}^{\prime} d x_{3}^{\prime}+C_{I 3 K 2}^{\mathrm{i}} \int_{-h}^{h} \tilde{u}_{K, 1}^{\prime} d x_{3}^{\prime}-\tilde{\beta}_{I 3}^{\prime \mathrm{i}} \int_{-h}^{h} \theta d x_{3}^{\prime} .
\end{aligned}
$$

According to the mean value theorem,

$$
\begin{aligned}
& \int_{-h}^{h} h_{3}^{\prime} d x_{3}^{\prime}=2 h \cdot h_{3}^{\text {avr }} \\
& \quad \approx h(\mathbf{y})\left[h_{n}^{\mathrm{i}}(h)-h_{n}^{\mathrm{i}}(-h)\right]=h(\mathbf{y}) \Delta h_{n}(\mathbf{y}), \\
& \int_{-h}^{h} \theta d x_{3}^{\prime}=2 h \cdot \theta^{\mathrm{avr}} \\
& \quad \approx h(\mathbf{y})[\theta(h)+\theta(-h)]=h(\mathbf{y}) \Sigma \theta(\mathbf{y}) \\
& \int_{-h}^{h} \tilde{\sigma}_{I 3}^{\prime} d x_{3}^{\prime}=2 h \tilde{\sigma}_{I 3}^{\prime a v r} \\
& \quad \approx h(\mathbf{y})\left[\tilde{t}_{I}^{\mathrm{i}}(h)-\tilde{t}_{I}^{\mathrm{i}}(-h)\right]=h(\mathbf{y}) \Delta \tilde{t}_{I}^{\prime}(\mathbf{y})
\end{aligned}
$$

For simplification of the model one can withdraw the interac- tion of thermo-magneto-electro-mechanical fields in the directions normal and tangential to the inclusion's median surface (as in the model of Winkler elastic foundation) by assuming that

$\int_{-h}^{h} \theta_{, 1}^{\prime} d x_{3}^{\prime}=0, \int_{-h}^{h} \theta_{, 2}^{\prime} d x_{3}^{\prime}=0 ;$

$\int_{-h}^{h} \tilde{u}_{K, 1}^{\prime} d x_{3}^{\prime}=0, \int_{-h}^{h} \tilde{u}_{K, 2}^{\prime} d x_{3}^{\prime}=0$.

In this case it is also assumed that

$\Sigma h_{n}(\mathbf{y})=0, \Sigma \tilde{t}_{I}=0$,

which allows to satisfy balance equations (1) identically.

Then, according to Eqs. (6)-(9), one can obtain

$$
\begin{aligned}
& \Delta h_{n}(\mathbf{y})=\frac{k_{11}^{\prime \mathrm{i}}(\mathbf{y})}{h(\mathbf{y})} \Delta \theta(\mathbf{y}), \\
& \Delta \tilde{t}_{I}^{\prime}(\mathbf{y})=-\frac{C_{I 3 K 3}^{\mathrm{i}}(\mathbf{y})}{h(\mathbf{y})} \Delta \tilde{u}_{K}^{\prime}(\mathbf{y})-\tilde{\beta}_{I 3}^{\prime \mathrm{i}} \Sigma \theta(\mathbf{y}) .
\end{aligned}
$$

Transforming (11) to global coordinates the following model of he thin thermomagnetoelectroelastic inclusion is obtained

$$
\begin{aligned}
& \Delta h_{n}(\mathbf{y})=\frac{k_{11}^{\prime \mathrm{i}}(\mathbf{y})}{h(\mathbf{y})} \Delta \theta(\mathbf{y}) \\
& \Delta \tilde{\mathbf{t}}(\mathbf{y})=-\frac{\mathbf{V}(\mathbf{y})}{h(\mathbf{y})} \Delta \tilde{\mathbf{u}}(\mathbf{y})-\mathbf{v}(\mathbf{y}) \Sigma \theta(\mathbf{y}) .
\end{aligned}
$$

Here $\mathbf{V}=\boldsymbol{\Omega}^{\mathrm{T}} \mathbf{C}_{33}^{\prime \mathrm{i}} \boldsymbol{\Omega}, \mathbf{v}=\boldsymbol{\Omega}^{\mathrm{T}} \tilde{\boldsymbol{\beta}}_{3}^{\prime \mathrm{i}}$.

Reducing Eqs (4), (5), (10), (12) one obtains the sought system of integral equations for a solid containing thin soft semipermeable shell-like inclusion

- heat conduction

$$
\begin{aligned}
& \frac{k_{11}^{\prime \mathrm{i}}}{2 h} \Delta \theta\left(\mathbf{x}_{0}\right)= \\
& \quad-\mathrm{HFP} \iint_{S} H_{i}^{* * *}\left(\mathbf{x}, \mathbf{x}_{0}\right) n_{i}\left(\mathbf{x}_{0}\right) \Delta \theta(\mathbf{x}) d S(\mathbf{x}), \\
& -\mathrm{TMEE} \\
& -\frac{V_{I K}\left(\mathbf{x}_{0}\right)}{2 h\left(\mathbf{x}_{0}\right)} \Delta \tilde{u}_{K}(\mathbf{y})= \\
& \quad n_{j}\left(\mathbf{x}_{0}\right)\left[-\operatorname{HFP} \iint_{S} S_{I j K}\left(\mathbf{x}, \mathbf{x}_{0}\right) \Delta \tilde{u}_{K}(\mathbf{x}) d S(\mathbf{x})\right. \\
& \left.\quad+\mathrm{CPV} \iint_{S}\left[Q_{I j}-H^{*}\left(\mathbf{x}, \mathbf{x}_{0}\right) v_{I}\left(\mathbf{x}_{0}\right)\right] \Delta \theta(\mathbf{x}) d S(\mathbf{x})\right] .
\end{aligned}
$$

Thus the problem is reduced to determination of unknown temperature and extended displacement discontinuities from the system of integral equations (13), (14). It should be mentioned that for the sake of compactness the terms accounting for external boundaries of the solid (which thermo-magneto-electromechanical loading is assumed to be given) are not written here. The explicit expressions for integral equations of external problem accounting for external boundaries of a solid can be found in (Pasternak et al., 2017). These terms are just the regular integrals, and for infinite medium they can be reduced to certain spatial functions. 


\section{NUMERICAL SOLUTION OF OBTAINED EQUATIONS}

Since the singularities of obtained kernels are the same as for 3D cracks, the solution strategy is the same as presented in (Pasternak et al., 2017). The median surface of thin inclusion is meshed with quadrilateral discontinuous boundary elements. Special shape functions are used to catch the square-root singularity, which also arises at inclusions front (see Sulym (2007)). Numerical evaluation of singular and hypersingular integrals is held with the help of modified Kutt's quadrature. Polynomial mappings are used to smoothen the integrand.

According to Sulym (2007) the field intensity factors vector at some point of inclusion's front is defined as

$\tilde{\mathbf{k}}^{(1)}=\lim _{\mathbf{x} \rightarrow \mathbf{x}(A)} \sqrt{\frac{\pi}{8 s(\mathbf{x})}} \mathbf{L} \cdot \Delta \tilde{\mathbf{u}}^{*}(\mathbf{x})$,

where $\tilde{\mathrm{k}}^{(1)}=\left[\mathrm{K}_{\mathrm{II}}, \mathrm{K}_{\mathrm{I}}, \mathrm{K}_{\mathrm{III}}, \mathrm{K}_{\mathrm{D}}, \mathrm{K}_{\mathrm{B}}\right]^{\mathrm{T}} ; \mathrm{K}_{\mathrm{I}}, \mathrm{K}_{\mathrm{II}}, \mathrm{K}_{\mathrm{III}}$ are the stress intensity factors; $K_{D}, K_{B}$ are electric displacement and magnetic induction intensity factors; $\mathrm{L}$ is a Barnett - Lothe tensor evaluated in the local coordinate system; and $\mathrm{s}(\mathrm{x})$ is an arc length evaluated from $\mathrm{x}$ to the selected point along the cross section of the crack with the normal plane.

These field intensity factors for soft semi-permeable inclusions are close to those of a crack; therefore, they can be accurately determined numerically by the technique proposed by Pasternak et al. (2017).

\section{NUMERICAL EXAMPLE}

Consider a transversely isotropic pyroelectric medium, which has the following properties of barium titanate (BaTiO3) (Dunn, 1993):

- elastic moduli (GPa): $C_{11}=C_{22}=150 ; \quad C_{33}=146$; $C_{12}=C_{13}=C_{23}=66 ; \quad C_{44}=C_{55}=44 ; \quad C_{66}=$ $\left(C_{11}-C_{12}\right) / 2=42$;

- piezoelectric constants $\left(\mathrm{C} / \mathrm{m}^{2}\right): \quad e_{31}=e_{32}=-4.35$; $e_{33}=17.5 ; e_{15}=e_{24}=11.4$;

- dielectric constants $(\mathrm{nF} / \mathrm{m}): \kappa_{11}=\kappa_{22}=9.86775 ; \kappa_{33}=$ 11.151;

- heat conduction coefficients $(\mathrm{W} /(\mathrm{m} \cdot \mathrm{K})): k_{11}=k_{22}=k_{33}=$ 2.5 ;

- thermal expansion coefficients $\left(\mathrm{K}^{-1}\right): \alpha_{11}=\alpha_{22}=8.53$. $10^{-6} ; \alpha_{33}=1.99 \cdot 10^{-6}$

- pyroelectric constants $(\mathrm{GV} /(\mathrm{m} \cdot \mathrm{K})): \lambda_{3}=13.3 \cdot 10^{-6}$;

- the rest of constants are equal to zero.

Here the Voigt notation is used in the indices of elastic moduli and piezoelectric constants, which changes the index pairs in Eq (3) with a single index as $11 \leftrightarrow 1 ; 22 \leftrightarrow 2 ; 33 \leftrightarrow 3 ; 23,32 \leftrightarrow$ $4 ; 13,31 \leftrightarrow 5 ; 12,21 \leftrightarrow 6$.

Consider the thermoelectroelastic problem for a pennyshaped disk inclusion of radius $\mathrm{R}$, which lays in the isotropy plane $O \mathrm{x}_{1} \mathrm{x}_{2}$. The medium is loaded with uniform heat $\mathrm{h}_{0}$ flowing along the polarization direction $\mathrm{Ox}_{3}$, which does not cause tertiary pyroelectricity (Pasternak et al., 2014). The median surface of inclusion is meshed with only 12 quadrilateral discontinuous boundary elements (Fig. 2).

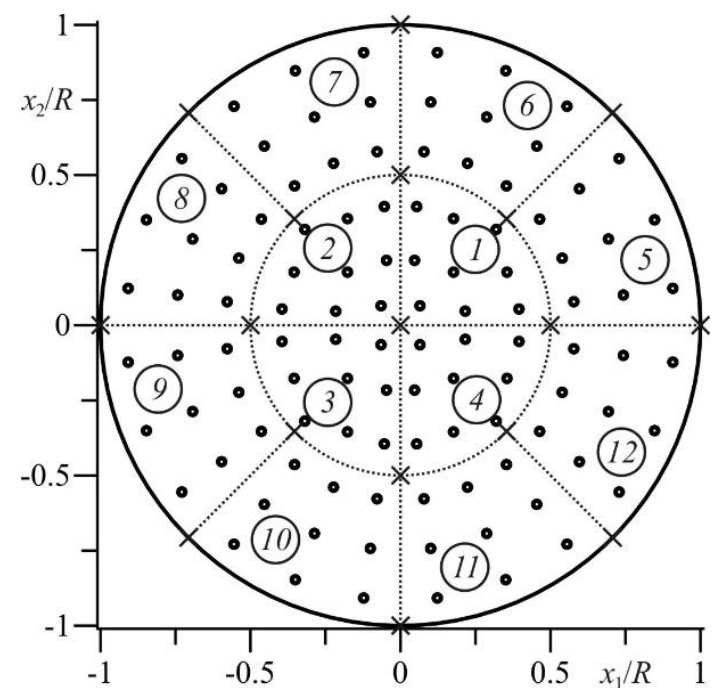

Fig. 2. Boundary element mesh of the disk inclusion

Four central boundary elements Nos 1-4 use general quadratic shape functions, while other elements (Nos 5-12) utilize special shape functions to account for the square root singularity of stress and heat flux at the inclusion front (Pasternak et al., 2017).

Inclusion's material is characterized by its relative rigidity, permeability and thermal conductivity, which are assumed the same in this study, i.e. $C_{33}^{\mathrm{i}}=r C_{33}, \kappa_{33}^{\mathrm{i}}=r \kappa_{33}, k_{33}^{\mathrm{i}}=r k_{33}$, where $\mathrm{r}$ is the dimensionless parameter. The thickness of inclusion is equal to $2 h=0.02 R$. It is also assumed that the plane $0 \mathrm{x}_{1} \mathrm{x}_{2}$ has zero temperature (with respect to the reference one). The normalization factors are equal to $K_{\sigma}=h_{0} \beta_{11} R \sqrt{\pi R} / k_{11}$, $K_{h 1}=-2 h_{0} \sqrt{R / \pi}$.

Fig. 3 depicts normalized stress intensity factors at inclusion's front for very small values of parameter $r<10^{-4}$. They are close to those calculated for a thermally insulated impermeable crack by Pasternak et al. (2017), which validates proposed model of thin shell-like soft weakly permeable inclusion.

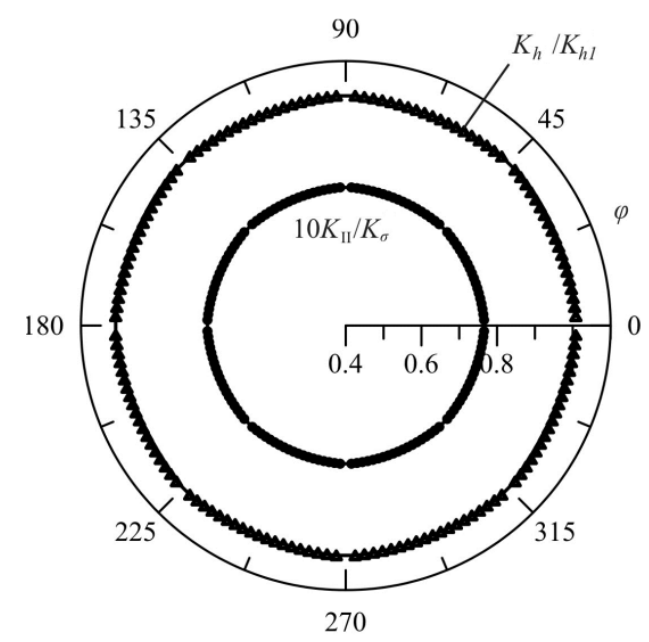

Fig. 3. Field intensity factors of a very soft inclusion

Fig. 4 depicts change in field intensity factor $\mathrm{K}_{\mathrm{II}}$ depending on the parameter $r$. The normalization factor is $\mathrm{K}_{\mathrm{II}}$ of a corresponding penny-shaped crack. 


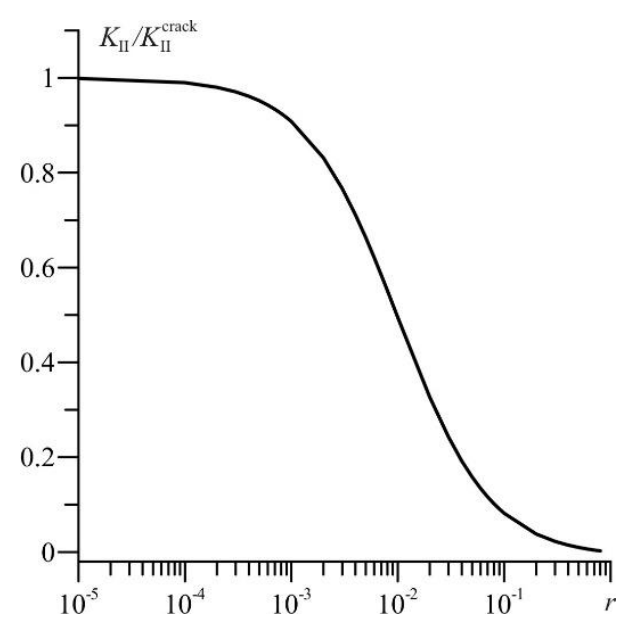

Fig. 4. Dependence of field intensity factor on $r$

One can see that the field intensity factor decrease monotonously on $r$. It is also visible that the proposed inclusion model does not sufficiently describe rigid inclusions. For $r>1$ field intensity factors and thus discontinuity functions are zero, the same as for a homogeneous material without inclusion. Therefore, another models should be developed to address this case.

\section{REFERENCES}

1. Aliabadi M.H. (1997) Boundary element formulations in fracture mechanics, Applied Mechanics Review, 50, 83-96.

2. Dunn M.L. (1993) Micromechanics of coupled electroelastic composites: effective thermal expansion and pyroelectric coefficients, J Appl Phys, 73, 5131-5140.

3. Muñoz-Reja M.M., Buroni F.C., Sáez A., García-Sánchez F. (2016) 3D explicit-BEM fracture analysis for materials with anisotropic multifield coupling, Applied Mathematical Modelling, 40, 2897-2912.
4. Mykhas'kiv V.V., Khay O.M., Zhang Ch., Bostrom A. (2010) Effective dynamic properties of 3D composite materials containing rigid penny-shaped inclusions, Waves in Random and Complex Media, 20(3), 491-510.

5. Pan E., Yuan F.G. (2000) Boundary element analysis of threedimensional cracks in anisotropic solids, Int. J. Numer. Meth. Engng., 48, 211-237.

6. Pasternak la., Pasternak R., Pasternak V., Sulym H. (2017) Boundary element analysis of $3 \mathrm{D}$ cracks in anisotropic thermomagnetoelectroelastic solids, Engineering Analysis with Boundary Elements, 74, 70-78.

7. Pasternak la., Pasternak R., Sulym H. (2014) Temperature field and heat flux that do not induce stress and electric displacement in a free thermoelectroelastic anisotropic solid, Mechanics Research Communications, 57, 40-43.

8. Rungamornrat J., Phongtinnaboot W., Wijeyewickrema A.C. (2015) Analysis of cracks in 3D piezoelectric media with various electrical boundary conditions, International Journal of Fracture, 192, 133-153.

9. Selvadurai A.P.S. (2000) An inclusion at a bi-material elastic interface, Journal of Engineering Mathematics, 37, 155-170.

10. Selvadurai A.P.S. (2002) Mechanics of a rigid circular disc bonded to a cracked elastic half-space, Int. J. of Solids and Structures, 39, 6035-6053.

11. Sulym H.T. (2007) Bases of mathematical theory of thermoelastic equilibrium of deformable solids with thin inclusions, Research and Publishing center of NTSh, Lviv, (in Ukrainian).

The present paper is financially supported by the Ministry of Science and Higher Education of Poland (research project No S/WM/4/2017) and realized in Bialystok University of Technology. 